\section{EIN NEUER MEDIZINISCHER ANSCHLUSSAPPARAT, DER SPANNUNGSTEILER , WIGOSTAT". Von}

Dr. R. Pophal.

An einen brauchbaren elektromedizinischen Apparat müssen folgende Anforderungen gestellt werden: I. Der Apparat muB konstanten galvanischen und einwandfreien faradischen Strom liefern; außerdem muß er für Endoskopie, Iontophorese und Kaustik eingerichtet sein; 2 . der Apparat muß leicht transportabel sein 3. Ströme, welche für den menschlichen Körper auch nur irgendwie gefährlich werden können, wie der Sinusstrom, dürfen nicht entnommen werden können; 4. der Apparat muB exakt arbeiten, völlig zuverlässig und handlich sein; 5. der Apparat muß möglichst billig sein. Gerade auf diesen letzten Punkt ist unter den heutigen Verhältnissen besonderer Wert zu legen.

Den eben genannten Forderungen entspricht, wenn auch nicht vollständig, so doch in weitgehendem Maße ein neuer kleiner Apparat, der unter der Bezeichnung Spannungsteiler (Wigostat) kürzlich in den Handel gebracht worden ist ${ }^{1}$ ). Auf das Prinzip des Spannungsteilers, der einen wesentlichen Fortschritt nicht allein für elektromedizinische $Z$ wecke, sondern für den gesamten Schwachstrombetrieb darstellt, wurde bereits vor Jahren von BRÜNINGS hingewiesen. Der Apparat besteht aus einem, auf einem isolierenden Hohlzylinder aufgewickelten, oxydierten und an den Kontak istellen blankgemachten Widerstandsdraht. Längs der beiderseitigen Kontaktstellen, die sich im mittleren Drittel des Drahtes befinden, können zwei Schleiffedern mit gegenläufigen Gewindehälften einander genähert oder von einander entfernt werden, so daß also die Länge des zwischen den beiden Schleiffedern abgegriffenen Widerstandsdrahtes kontinuierlich verändert werden kann. Ein Anschlag sorgt dafür, daß die beiden Federn nur bis zu einem gewissen Höchstbetrage voneinander entfernt werden können. Die beiden Federn sind metallisch mit zwei Klemmen, von welchen die Niederspannung entnommen wird, verbunden. Der Apparat stellt demnach einen Spannungstciler dar, aus dessen Mitte man gemäß seiner Ausführung beliebige Spannungswerte entnehmen kann. Diese Spannungswerte lassen sich allmählich innerhalb einer gewissen Grenze von $O, I$ Volt an aufwärts variieren. Der Apparat kann an Gleich-oder Wechselstromleitungen von IIO oder 220 Volt mittelst eines Steckkontaktes angeschlossen werden. Für seine medizinische Anwendung kommt bis auf wenige Aus nahmen GleichstromanschluB in Frage. Der Apparat ist stets gebrauchsfertig, auBerordentlich kompendiös und sehr leicht. Der Eigenverbrauch ist minimal. Bei Anschluß an eine Gleich- stromleitung kann der Spannungsteiler ohne weiteres zur Galvanisation benutzt werden. Die Elektroden können dabei direkt an die Niederspannungsklemmen angeschlossen werden, Ein Milliamperemeter ist selbstverständlich, wie zu jeder Galvanisatión, wünschenswert. Ebenso einfach ist die Verwendung des Spannungsteilers zur Endoskopie, Iontophorese, sowie für den Betrieb niedervoltiger Glühlampen, Klingelanlagen und für Laboratoriumszwecke. Da der Apparat eine höhere Dauerbelastung als 750 Milliampere nicht verträgt, so ist er für Kaustik nicht geeignet. Zur Erzeugung faradischen Stromes ist es nur nötig, an die Niederspannungsklemmen einen Schlitteninduktionsapparat anzuschlieBen.

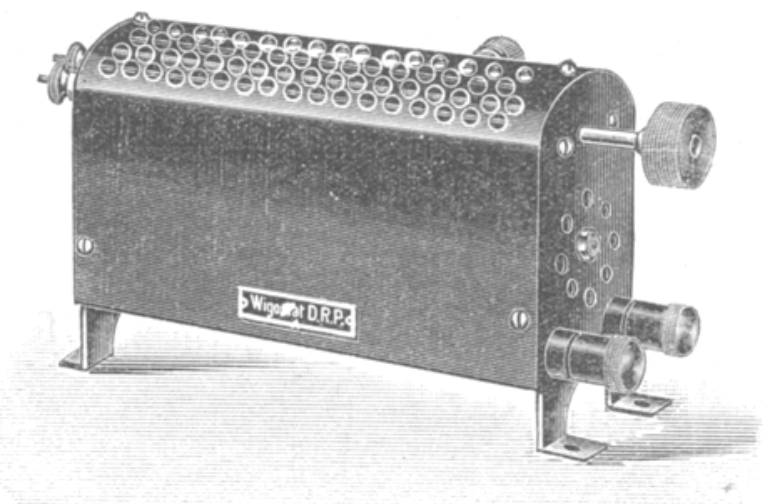

Da der Apparat völlig zuverlässig, sehr handlich, sehr leicht transportabel und auch verhältnismäßig billig ist; so erscheint er für elektromedizinische Zwecke besonders geeignet. $\mathrm{Er}$ kann ohne Schwierigkeiten in der Tasche mit in die Wohnung des Patienten genommen und dort nach Anschluß an eine Steckdose zur galvanischen Untersuchung oder zu endoskopischen Zwecken (Cystoskop, Stirnlampe) gebraucht werden. Ein Ubergang des Starkstromes in die Niederspannungsleitung kann nicht erfolgen. "Seibst Kurzschlu $B$ in letzterer ist ungefährlich. Der Apparat kann auch zusammen mit einem Schlitteninduktionsapparat, Volt- und Milliamperemeter, Stromwender und Stromwechsler, zweckmäßig auf einer kleinen Schálttafel montiert, so daß sich ein jedesmaliges Zusammenschalten erübrigt, von der vorher genannten Firma bezogen werden. (Aus der Psychiatrischen- und Nervenklinik der Universität Greifswald [Direktor Prof. Schröder].)

\title{
NEUE SPEZIALITÄTEN (einschl. Nährpräparate und Geheimmittel).
}

Die Angaben über Zusammensetzung und Indikation stammen unmittelbar oder mittelbar von den produzierenden Firmen, soweit nicht ausdrücklich ein Autor oder ein Institut als Unterschrift genannt ist.

Augenfiuer (Schöne Augen für ,Augenfeuer") soll laut Gebrauchsanweisung , den Augen wunderbares Feuer geben und sie ausdrucksvoll machen". Die Wirkung soll eine nachhaltige sein. Die von Dr. Aufrecht, Berlin NW 6, vorgenommene qualitative und quantitative Analyse ergab, daß in $100 \mathrm{ccm}$ der Flüssigkeit enthalten waren: $\mathrm{B} 2 \mathrm{i}$ I $00^{\circ} \mathrm{C}$ flüchtige Stoffe $97,08 \mathrm{~g}$, organische (durch Alkohol fällbare) Stoffe I,88, Borsäure 1,04 g. Das Mittel soll danach wahrscheinlich eine wässerig schleimige, mittels Borsäure konservierte Abkochung von Carragheen sein ${ }^{2}$ ).

Gallensfeinkur, ein Mittel des ,Heilkundigen" OfFrRmanN in Köln a. Rh. besteht aus drei Flüssikeiten. Auf Veranlassung der Geschäftsstelle des D. Ap.-V. wurden diese Präparate im pharmaz. Inst. der Universität Frankfurt a. M. von C. A. RojAHN untersucht. Bei dem Präparat I handelt es sich um den Auszug einer emodinhaltigen Droge, vermutlich um einen Sennaaufguß mit einem Zusatz von SüBholz. Der Inhalt der Flasche II scheint nur aus einem sehr dünnen Aufguß einer Minzenart zu bestehen. Flasche III enthält $46 \mathrm{~g}$ Ricinusöl, das mit einer rotgefärbten, wässerigen Flüssigkeit ohne Emulgierungsmittel vermischt ist ${ }^{3}$ ).
Matori"Austria-Tabletten, als , antikonzeptionell und antiseptisch" wirkendes Mittel vertrieben, bestehen nach einem Analysenergebnis von Dr. Aufrechr, Berlin NW 6, aus einer Mischung von Weinsäure, Borax, bzw. Natriumperborat, Natriumbicarbonat, Kochsalz und Talkum ${ }^{1}$.

Peerless-Hair-Removar, a reliable Safe Depilatory. Made by Peerless Cosmetics Co., New-York. Die quantitative Analyse (Dr. AUFRECHT) des Mittels ergab folgende Zusammensetzung: Unterschwefligsaures Natron . . . . . . . . . 68,70\% Kohlensaures Natron . . . . . . . . . . . . . . $26,42 \%$ Chlornatrium . . . . . . . . . . . . . . . . . . . . $4,88 \%$ Rhönisol ist nach einem Untersuchungsergebnis aus dem Forschungsinstitut für klinische Pharmakologie in Hamburg-Eppendorf die Auflösung eines sauren Kunstharzes, das beträchtliche Mengen freien Phenols enthält. Formaldehyd war weder frei noch gebunden nachzuweisen. Angepriesen wird das Mittel als Lösung von "Rhönitbalsam", der ein Kondensationsprodukt des Formaldehyds mit Karbolsäure sein soll, in Alkohol oder Aceton zur Verwendung bei parasitären Dermatosen, zum Desinfizieren und zum Verschluß von Wunden usw. D. Rhönit-Werk in Hamburg ${ }^{2}$ ).

\section{BRIEF AUS LETTLAND.}

\section{Die akuten Infektionskrankheiten in der Republik Lettland im Jahre I92t.}

Wenn man bedenkt, wie wir, bei der bolschewistischen Invasion I919 in einer Flut von Infektionskrankheiten. zu ertrinken

\footnotetext{
Erhällich rei M. Schlר@nagel Nürnberg, Steinbuhlerstr. 16. (Preis etwa 400 Mk.) Tharmaz, Ztg. I92I, $S$. IO5g.

3) Pharm. Zentralhalle I 921, S. 74 I
}

drohten, wenn man ferner in Trwägung zieht, daß dem neugebildeten Staate so gut wie alles fehlte, um den Kampf mit den Infektionskrankheiten erfolgreich aufzunchmen, muß man anerkennen,

\footnotetext{
1) Apolhektr-Z1g. I921, S. 474 .

8) Pharmaz. Ztg. I921, S. 1059.
} 
daß wir mit der Bilanz pro I92 I halbwegs zufrieden sein können. Man vergesse nicht die geographische Lage Lettlands, seine ausgedehnte Grenze mit dem verseuchten Sowjetkoloß, die noch wenig ausgebildete hygienische Erziehung eines nicht kleinen Teiles der Bevölkerung und verschiedenes andere und wird dann verstehen, daß wir doch mit ,osteuropäischen “ Morbiditäts- und Mortalitätsziffern rechnen müssen. In vieler Beziehung spielt Lettland die undankbare (und auch kostspielige) Rolle eines ,Seuchenfilters" gegenüber Westeuropa und vieles ist à conto dieser Aufgabe zu setzen.

Lettland hat eine Bevölkerung von ca. I 728000 Einwohnern, die auf einem verhältnismäßig sehr großen Areal wohnen.

Von den 4 Provinzen der Republik, Livland (das frühere Südlivland), Kurland, Semgallen und Lettgallen, ist letztere sehr dünn bevölkert und sehr stark verseucht. Es ist gerade die Provinz (früher auch Polnisch-Livland genannt), die an Rußland grenzt, eine wenig kultivierte (hauptsächlich katholische) Bevölkerung hat und auch sonst das "Wild-West" unserer Republik darstellt. Von hier aus findet häufig eine Verbreitung der Krankheiten statt.

Wenn wir uns nun den gemeingefährlichen Seuchen zuwenden, so können wir mit Befriedigung sagen, daß nicht ein einziger Fall von Pest und nur ein einziger (tödlicher) Fall von Cholera im Berichtsjahre zu verzeichnen waren. Letzterer Fall bezieht sichauf einen Rückwanderer, der am Tage seiner Ankunft krank ins Hospital geschafft wurde und dort bald darauf verstarb. Die bakteriologische Untersuchung des Dünndarminhaits ergab Choleravibrionen nahezu in Reinkultur (Choleratyphoid). Sämtliche mit dem Kranken in Berührung gekommenen Personen desselben Rückwanderertransports wurden isoliert und alle Maßnahmen gegen die Weiterverbreitung ergriffen. Es ist, entgegen allen Pressenachrichten in skandinavischen Blättern, kein anderer Fall von Cholera vorgekommen und ordnungsgemä $\beta$ wurde er allen konsularischen Vertretern gemeldet $\left.{ }^{1}\right)$. Wenn wir uns nun den Morbiditätsziffern der anderen Infektionskrankheiten zuwenden, so muß zunächst gesagt werden, daß alle Zahlen nur einen sehr bedingten Wert haben; auf dem flachen Lande und den kleinen Landstädten wird das Meldesystem nicht allzu streng gehandhabt. Am wenigsten gilt aber diese Einschränkung für das Fleckfieber, dessen Meldung streng vorgeschrieben ist ${ }^{2}$ ). Nur aus Lettgallen mögen einzelne Fälle unangemeldet geblieben sein, da hier die Zahl der Ärzte gering ist und Heilgehilfen (sog. Feldscher) die ärztliche Tätigkeit ausüben. Es sind für Lettland im Berichtsjähre im ganzen 1288 Fälle gemeldet worden, von denen ca. 90\% eingeschleppte Fälle sind. Das Gesundheitsamt hatte ein Rundschreiben an sämtliche Kreisärzte gerichtet, um genaue Daten über die Herkunft der einzelnen Fälle zu erhalten und da stellte sich diese Tatsache auf das evidenteste heraus. Die ïbrigen Io \% beziehen sich auf Angehörige der Erkrankten und Pflegepersonal. Erwähnt sei, daß der gesamte Litauische Rückwandererstrom sich gleichfalls durch Lettland ergießt, wa; unter anderem eine Zunahme der Erkrankungen in Dünaburg verursachte. Unsere Quarantäne-Station und Entlausungsanstalt in Rositten ist nicht in der Lage, die zuweilen sehr großen Rückwanderertransporte genügend $\mathrm{zu}$ versorgen, und leider müssen wir sagen, daB Litauen seinen Verpflichtungen gegenüber seinen Staatsangehörigen nicht immer nachkommt, so daß ärztlich ungenügend versorgte und verseuchte Transporte den langen Weg von Rositten über Dünaburg zur litauischen Grenze machen und Gelegenheit zu weiteren Ansteckungen geben. Auffallend ist die außerordentlich geringe Mortalität von $2 \%$, höchstens $5 \%$, auch bei den ausgehungerten und erschöpften Rückwanderern, und die geringe Kontagiosität. Das Fleckfieber hat für uns nahezu seinen Schrecken verloren. Wir wollen es dahingestellt bleiben lassen, ob eine Virulenzverminderung oder andere Ursachen eine Rolle spielen. Gewiß ist die Bevölkerung Lettlands zum größten Teil als läusefrei anzusehen (mit Ausnahme Lettgallens), aber auch die direkte Berïhrung mit den verlausten Kranken führt jetzt selten zur Ansteckung. Die Erfahrung mit unserer Quarantänestation in Rositten zeigt auch, daß das Hauptaugenmerls bei der Fleckfieberbekämpfung auf innere Maßnahmen zu richten ist, und sich auf Grenzstationen die Maßnahmen auf eine tunlichst sorgfältige Entlausung und Zurückhaltung der Kranken zu beschränken hat, ohne die kostspielige Beobachtung Anstekkungsverdächtiger. - Auch beim Recurrens (275 Meldungen)

1) Auch Ig2o haben wir nut einen Fall von Cholera zu verzeichnen gehabt. Det Fall, es handelte sich um einen Soldaten der Grenzwache, wurde gleichfalls von uns bakteriologisch untersucht.

2) Es ist selbstverständlich ausgeschlossen, daß Fälle von Pest oder Cholera be uns nicht gemeldet werden würden. Im Gagenteil, häufig exfolg sa vo. Ärzten Alarmnachrichten, die sich in der Folge als unrichtig erweisen. handelt es sich beinahe ausschließlich um Rückwanderer; nirgends ist es zu einer Weiterverbreitung der Seuche gekommen. Die Zahl der Erkrankten ist aber sicher eine viel größere. - Was die Pocken anbetrifft ( 254 M.), so spielen Rückwanderer hier eine weniger große Rolle als Erkrankte; die wenigen Erkrankten haben aber als Verbreiter der Seuche in Dörfern eine Rolle gespielt. Einen Impfzwang gibt es bei uns nicht. Als Kuriosum mag angeführt werden, daß aus Lettgallen nur ganz vereinzelte Fälle gemeldet worden sind, trotzdem dort zahlreiche altgläubige Russen leben, die aus religiösen Anschauungen die Impfung verweigern. Man sieht schon hieraus, wie wenig auf die Angaben aus Lettgallen etwas $z \mathfrak{u}$ geben. ist. Die aus Rußland eingeschleppten Pocken müssen als besonders bösartig angesehen werden, denn auffallend ist die Häufigkeit der hämorrhagischen Fälle (Purpura variolosa und Variola haemorrhag.). Ich habe allein in Riga in den letzten 3 Jahren I 3 derartige Fälle seziert. In anderen Gegenden Lettlands ist dasselbe beobachtet worden. Sie treten auch bei Geimpften auf (Impfnarben). - Was die Lepra anbetrifft, die wir wegen ihrer Gemeingefährlichkeit auch hier erörtern wollen, so weist das Jahr I 92 I die hohe Zahl von 29 Neumeldungen auf. Bekanntlich bildete Lettland gerade das Gebiet des ehemaligen russischen Reiches, das am lepraverseuchtesten war. I9I4 betrug die Zahl der Leprösen in Livland (inkl. den nördlichen, jetzt zur Republik Estland gehörigen Teil) 412, in Kurland 202. Im Januar 192 I hatte Lett. land 24.0 Lepröse, zu denen jetzt die 29 neuen Fälle hinzukommen. $\mathrm{Daß}$ die Ansteckungsmöglichkeiten während des Krieges und der Revolution gestiegen sind, liegt auf der Hand, da viele Lepröse die Leprosorien verließen. Beim Ausbruch der russischen Revolution setzten die Leprösen im Rigaschen Leprosorium ihren Arzt ab, erklärten sich für autonom und verübten allerlei anderen Unfug. Die Folgen davon werden wir wohl noch in späteren Jahren sehen. - Der Abdominaltyphus spielt sowohl in Riga, wie überall in Lettland, eine große Rolle ( 1363 MI.). In Riga ist es infizierte Milch, Bacillenträger und Dauerausscheider, die die Infektionen vermitteln, an der Peripherie der Stadt wohl auch infizierte Brunnen. Die Stadt selbst hat vorzügliche Wasserleitung und Kanalisation. Sehr häufig ist bei uns der Paratyphus $(B)$ abdominalis, häufiger, wie man auch hier vermutet (bakteriolog. Erfahrungen). I9I7 stellten wir bei 200 klinisch Typhuskranken i 8 Paratyphusfälle fest; dieses Verhältnis wird sich eher zugunsten des Paratyphús geändert haben. - Die Dysenterie hat sowohl in Riga, wie auch sonst im Lande nach dem Kriege eine starke Abnahme erfahren, jedoch ist die Zahl der Meldungen ( 1 162) vollkommen ungenau. In Riga herrscht die Shiga-Kruse-Form vor $(80 \%)$, die übrigen sind Y-Fälle. Flexner-Bacillen haben wir nur während des Krieges vereinzelt gefunden. - Die epidemische Genickstarre (8I M.) tritt bei uns nur sporadisch auf und hat nie zu ausgedehnten Epidemien geführt. Jedes Jahr haben wir Gelegenheit mehrere Fälle auf dem Obduktionstisch oder bakteriologisch zu sehen. - Morbilli (3072 M.), Scarlatina (I 522 M.) und Diphtherie (964 M.) bieten keinerlei epidemiologisches Interesse. - Die Influenza hat im verflossenen Jahre in der Epidemiologie unseres Landes gar keine Rolle gespielt. Erst jetzt beginnt wieder eine stärkere Ausbreitung (Januar 1922) und es sieht so aus, als ob wir wieder vor einer größeren Epidemie stehen. Influenzabacillen haben wir sowohl früher (1918) wie auch jetzt im Frühstadium im Nasensekret sehr häufig gefunden. - Die vielleicht mit der Influenza im Zusammenhang stehende Encephalitis lethargica ist bei uns auch nur ganz vereinzelt aufgetreten, immerhin aber häufiger wie es die Zahl der Meldungen ( 7 ) erscheinen läßt. - Ein immer größer werdendes Interesse erregt bei uns die Malaria, die, durch Rückwanderer eingeschleppt, eine immer größere Verbreitung findet. Die Malariaverseuchung der Rückwanderer ist ganz enorm, und da bei uns die Anopheles weit verbreitet ist, müssen wir auf der Hut sein. Das Gesundheitsamt hat die Ärte nachdrücklich auf ihre Pflicht aufmerksam gemacht, Malariafälle zu melden, was aber nur zu einem kleinen Bruchteils (Ig2 Fälle) geschehen ist. Die außerordentlich interessanten Beo jachtungen, die wir in der Malariafrage gemacht haben, werden wir an anderer Stelle mitteilen.

Zum SchluB geben wir noch die Mortalitätsziffern der wichtigsten akuten Infektionskrankheiten bei der Bevölkerung Rigas (ca. 260000 Einwohner) für das Jahr I921 wieder: Es verstarben an Bauchtyphus 35, Fleckfieber 40, Cholera I, Recurrens 8, Variola 24. Influenza 19, Dysenterie 53.

Vergleicht man diese Zahlen mit denen während des Krieges, wo wir z. B. I9I7 2022 Todesfälle an Dysenterie, I9I9 154I an Fleckfieber, 458 an Pocken usw. in Riga zu verzeichnen hatten, so wird man mit den jetzigen Zahlen zufrieden sein können. ${ }^{1}$ ) R. ADELHEIM.

1) Alle angeführten Zahlen sind amtlichem Material entnommen. 